\title{
Separating Convex Sets in the Plane
}

\author{
Jurek Czyzowicz, ${ }^{1}$ Eduardo Rivera-Campo, ${ }^{2}$ Jorge Urrutia, ${ }^{3}$ \\ and Josepth Zaks ${ }^{4}$ \\ ${ }^{1}$ Département d'Informatique, Université du Québec à Hull, \\ Hull, Québec, Canada \\ ${ }^{2}$ Departamento de Matemáticas, Universidad Autónoma Metropolitana-I, \\ México D.F., México \\ ${ }^{3}$ Department of Computer Science, University of Ottawa, \\ Ottawa, Ontario, Canada \\ ${ }^{4}$ Department of Mathematics, University of Haifa, Haifa, Israel
}

\begin{abstract}
Given a set $A$ in $R^{2}$ and a collection $S$ of plane sets, we say that a line $L$ separates $A$ from $S$ if $A$ is contained in one of the closed half-planes defined by $L$, while every set in $S$ is contained in the complementary closed half-plane.

We prove that, for any collection $F$ of $n$ disjoint disks in $R^{2}$, there is a line $L$ that separates a disk in $F$ from a subcollection of $F$ with at least $\Gamma(n-7) / 4\rceil$ disks. We produce configurations $H_{n}$ and $G_{n}$, with $n$ and $2 n$ disks, respectively, such that no pair of disks in $H_{n}$ can be simultaneously separated from any set with more than one disk of $H_{n}$, and no disk in $G_{n}$ can be separated from any subset of $G_{n}$ with more than $n$ disks.

We also present a set $J_{m}$ with $3 m$ line segments in $R^{2}$, such that no segment in $J_{m}$ can be separated from a subset of $J_{m}$ with more than $m+1$ elements. This disproves a conjecture by $\mathrm{N}$. Alon et al. Finally we show that if $F$ is a set of $n$ disjoint line segments in the plane such that they can be extended to be disjoint semilines, then there is a line $L$ that separates one of the segments from at least $\lceil n / 3\rceil+1$ elements of $F$.
\end{abstract}

\section{Introduction}

Given a collection $F$ of disjoint compact convex sets in the plane, an element $A \in F$, and a subcollection $S$ of $F$, we say that a line $L$ separates $A$ from $S$ if $A$ is contained in one of the closed half-planes defined by $L$, while every set in $S$ is contained in the complementary closed half-plane. 
In [3] Tverberg proves that, for any positive integer $k$, there is a minimum integer $N(k)$ such that in any family $F$ of $N(k)$ or more disjoint compact convex sets in the plane there is one that can be separated from a subfamily with at least $k$ sets. In [2] Hope and Katchalski prove that $3 k-1 \leq N(k) \leq 12(k-1)$.

In this article we show that, for any collection $F$ of $n$ disjoint disks in $R^{2}$, there is a line $L$ that separates a disk in $F$ from a subcollection of $F$ with at least $\Gamma(n-7) / 47$ disks. We produce configurations $H_{n}$ and $G_{n}$ with $n$ and $2 n$ disks, respectively, such that no pair of disks in $H_{n}$ can be simultaneously separated from any set with more than one disk of $H_{n}$; and no disk in $G_{n}$ can be separated from any subset of $G_{n}$ with more than $n$ disks.

In Section 3 we present a configuration $J_{m}$ with $3 m$ line segments in $R^{2}$, such that no segment in $J_{m}$ can be separated from a subset of $J_{m}$ with more than $m+1$ elements. This disproves a conjecture by Alon et al. presented in [1]. Finally, we show that if $F$ is a collection of $n$ line segments such that they can be extended to be disjoint semilines, then there is a line $L$ that separates one of the segments from a subcollection of $F$ with at least $\Gamma(n+3) / 3\rceil$ elements.

The results in this article remain valid for corresponding collections of convex sets with pairwise disjoint relative interiors. This allows us to present, as examples, the configurations $H_{n}, G_{n}$, and $J_{m}$ that contain sets with common boundaries but pairwise disjoint relative interiors.

\section{Separating Disks}

In [1], Alon et al. proved that there is a constant $c>0$ such that, for any family $F$ with $n$ disjoint congruent disks, there is a line $L$ that leaves at least $k / 2-c \sqrt{k} \sqrt{\log k}$ disks on each closed half-plane defined by $L$. When the disks are allowed to have arbitrary radii the situation is entirely different as the following example illustrates.

We describe a configuration $H_{n}$ of $n$ disks in which no pair $C_{i}, C_{j}$ of disks in $H_{n}$ can be simultaneously separated by one line $L$ from any other pair $C_{k}, C_{l}$ in $H_{n}$.

Let $S_{1}>S_{2}>\cdots>S_{n}$ be $n$ different slopes such that $0 \leq S_{i} \leq \varepsilon$, with $\varepsilon$ small enough. Let $H_{n}$ consist of $n$ disks defined recursively as follows:

(a) $C_{1}$ is any disk in $R^{2}$.

(b) $C_{i+1}$ is a disk tangent to $C_{i}$ such that the slope of the line that separates them is $S_{i}$.

(c) $C_{i+1}$ is large enough such that any line $L$ separating $C_{j}$ from $C_{i+1}$, $1 \leq j<i+1$, has slope $s(L)$ contained in the interval $\left(S_{i}-\delta, S_{i}+\delta\right), \delta>0$, $\delta$ much smaller than $\varepsilon$. Observe that $s(L)$ is contained in the interval $(-\delta, \varepsilon+\delta)$ since $0 \leq S_{i} \leq \varepsilon$.

Moreover, if $\delta$ is small enough, $C_{i+1}$ can be chosen such that:

(d) Any line separating $C_{j}$ from $C_{i}, 1 \leq j<i$, intersects $C_{i+1}$.

It follows that there are no different pairs of disks $\left\{C_{i}, C_{j}\right\}$ and $\left\{C_{k}, C_{l}\right\}$ in $H_{n}$, such that there is a line separating $\left\{C_{i}, C_{j}\right\}$ from $\left\{C_{k}, C_{l}\right\}$. For let us assume that 
$i$ is the smallest of $i, j, k$, and $l$, and that $k<l$. It now follows from (d) that any line separating $C_{i}$ from $C_{k}$ must intersect $C_{l}$.

Notice that in $H_{n}, C_{i}$ can be separated from $C_{1}, \ldots, C_{i-1}, i=1, \ldots, k$, and that $C_{i}$ cannot be separated from any pair $C_{k}, C_{l}, i<k<l$.

For any family of disjoint disks we have the following theorem:

Theorem 1. In any family $F$ of $n$ disjoint disks, there is one disk that can be separated from a subfamily of $F$ with at least $\lceil(n-7) / 4\rceil$ disks.

The following lemma will be used in the proof; the reader may wish to verify it.

Lemma 2. Let $H$ be a family of $m$ disjoint disks, all of which are intersected by two orthogonal lines. There is a disk in $H$ that can be separated from a subfamily of $H$ with at least $\lceil(m-5) / 2\rceil$ disks.

Proof of Theorem 1. Sweep a vertical line $L_{1}$, from left to right, until one disk is left to the left of $L_{1}$. Then sweep a horizontal line $L_{2}$, from bottom to top, until a disk is left below $L_{2}$. Let $n_{1}$ and $n_{2}$ denote the number of disks to the right of $L_{1}$ and above $L_{2}$, respectively. Also let $H$ be the set of disks in $F$, intersected by both $L_{1}$ and $L_{2}$ and denote by $n_{3}$ the number of disks in $H$. Clearly, $n_{3} \geq n-n_{1}-n_{2}-2$.

By Lemma 2, there is a disk in $H$ that can be separated from a subfamily with at least $\left\lceil\left(n_{3}-5\right) / 2\right\rceil$. If $n_{1}<\lceil(n-7) / 4\rceil$ and $n_{2}<\lceil(n-7) / 4\rceil$, then $\left\lceil\left(n_{3}-5\right) / 2\right\rceil \geq$ $\Gamma(n-7) / 4\rceil$ and the result follows.

The following example shows that, occasionally, we cannot separate any disk of a family of $m$ disks from any subfamily with more than $m / 2$ disks.

To construct the family $G_{n}$ let us take a copy $H_{n}^{\prime}=\left\{C_{1}^{\prime}, C_{2}^{\prime}, \ldots, C_{n}^{\prime}\right\}$ of the configuration $H_{n}$ as follows: reflect $H_{n}$ along the $x$-axis and translate it in the northwest direction until all the lines separating $C_{i}$ from $C_{j}$ intersect only $C_{n}^{\prime}$ in $H_{n}^{\prime}$ and all lines separating $C_{i}^{\prime}$ from $C_{j}^{\prime}$ intersect only $C_{n}$ in $H_{n}$ (see Fig. 1).

Any line separating two elements $C_{i}, C_{j}$ in $H_{n}$ leaves at most $C_{1}, \ldots, C_{i}$ on one side and $C_{1}^{\prime}, \ldots, C_{n-1}^{\prime}$ on the other; similarly, for any line separating two elements in $H_{n}^{\prime}$. Then $G_{n}$ is a configuration with $2 n$ disks and none of them can be separated from any set of disks in $G_{n}$ with more than $n$ disks.

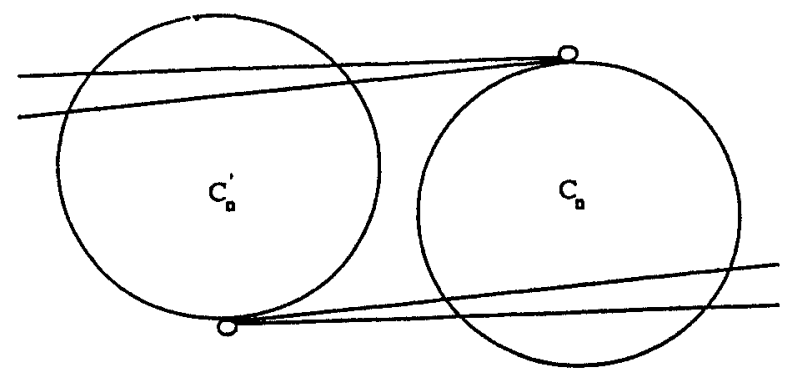

Fig. 1. $C_{1}, C_{2}, \ldots, C_{n-1}$ are contained in a small circle above $C_{n}$. 


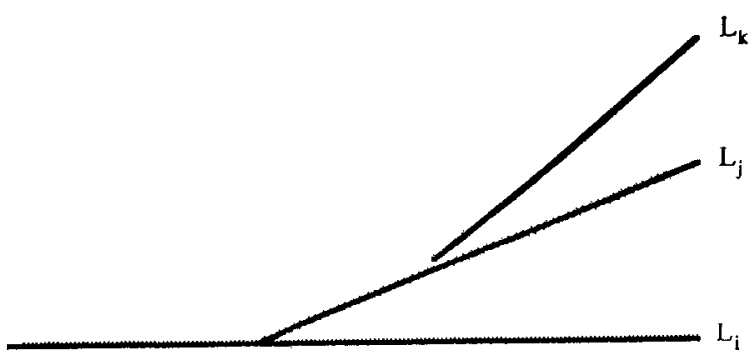

Fig. 2

\section{Separating Line Segments and Semilines}

In [1] the following conjecture is presented: for any collection $F$ of $n$ disjoint line segments on the plane, there is an element $S$ of $F$ that can be separated from close to $n / 2$ elements of $F$. In this section we disprove the conjecture by producing a family $J_{m}$ of $3 m$ line segments such that no element of $J_{m}$ can be separated from more than $m+1$ elements of $J_{m}$.

To describe $J_{m}$ we use a configuration due to K. P. Villanger, see [3]. He constructs a family $T$ of $m$ line segments $L_{1}, L_{2}, \ldots, L_{m}$ with the property that, for each $k=3, \ldots, m, L_{k}$ intersects the convex closure of $L_{i} \cup L_{j}, 1 \leq i<j<k$, and therefore $L_{k}$ cannot be separated by a line from $\left\{L_{i}, L_{j}\right\}$ (see Fig. 2).

His construction may be reproduced in such a way that $L_{1}, L_{2}, \ldots, L_{m}$ have slopes $0=S\left(L_{1}\right)<S\left(L_{2}\right)<\cdots<S\left(L_{m}\right)=\delta<\pi / 2$, respectively; and such that, for $i=1,2, \ldots, m$, the left endpoint of $L_{i+1}$ lies in an interior point of $L_{i}$ within distance $\varepsilon$ of the left endpoint of $L_{1}$ (see Fig. 3).

Our example is a set $J_{m}$ of $3 m$ line segments consisting of three copies $T_{0}=\left\{L_{0,1}, \ldots, L_{0, k}\right\}, T_{1}=\left\{L_{1,1}, \ldots, L_{1, k}\right\}$, and $T_{2}=\left\{L_{2,1}, \ldots, L_{2, k}\right\}$ of $T$ placed around a triangle $Q$ with vertices $v_{0}, v_{1}, v_{2}$ (see Fig. 4). The values of $\varepsilon$ and $\delta$ are chosen in such a way that the line supporting any element of $T_{i}$, intersects all the elements of $T_{i+1}$; addition taken mod 2.

Let us consider the case where the segments in $F$ can be extended to semilines so that they remain pairwise disjoint.

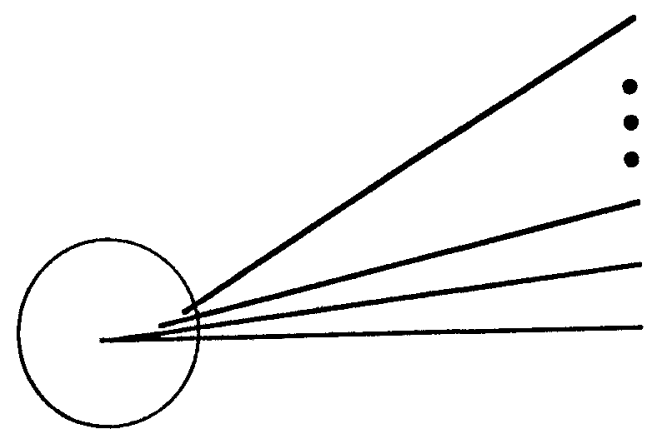

Fig. 3 


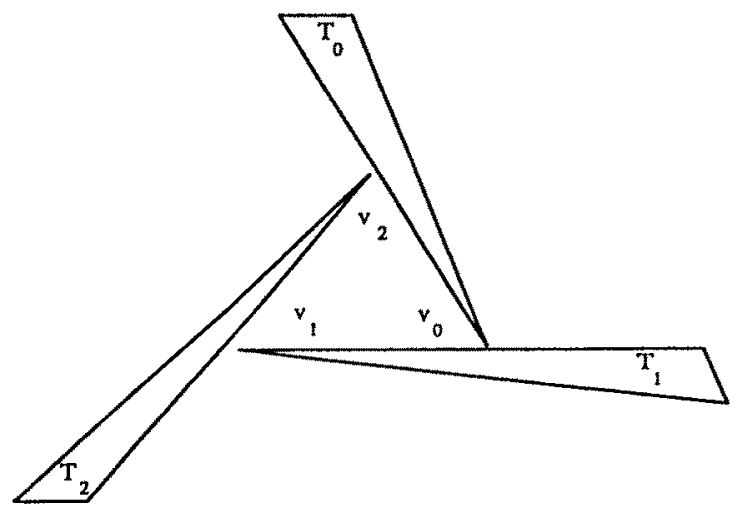

Fig. 4

Theorem 3. Let $F=\left\{L_{1}, \ldots, L_{n}\right\}$ be a family of $n$ disjoint line segments, $n \geq 4$. If they can be extended to form a collection of disjoint semilines, then there is a line $L$ that separates an element $L_{i}$ of $F$ from a subset of $F$ with at least $\lfloor n / 3\rfloor+1$ elements.

Proof. If there is an element $L_{i}$ of $F$ that can be extended to a whole line without intersecting any other element of $F$, then $L_{i}$ can be separated from a subfamily of $F$ with at least $\lceil(n-1) / 2\rceil$ elements of $F$. Suppose then that the line supporting each $L_{i}$ intersects at least another element $L_{i}$ of $F$. Extend the elements of $F$ as much as possible until a family $F^{\prime}=\left\{L_{1}^{\prime}, \ldots, L_{n}^{\prime}\right\}$ of semilines is obtained such that;

1. The endpoint of every element of $F^{\prime}$ lies on an interior point of another element of $F^{\prime}$.

2. No two elements of $F^{\prime}$ cross each other (see Fig. 5).

We say that $L_{i}^{\prime}$ hits $L_{j}^{\prime}$ if the endpoint of $L_{i}^{\prime}$ lies on $L_{j}^{\prime}$. For example, in Fig. 5 $L_{1}^{\prime}$ hits $L_{4}^{\prime}$. It is easy to see that in $F^{\prime}$ there is a cyclic sequence of elements, say $L_{1}^{\prime}, \ldots, L_{j}^{\prime}, j \leq n$, such that $L_{i+1}^{\prime}$ hits $L_{i}^{\prime}, i=1, \ldots, j-1$, and $L_{1}^{\prime}$ hits $L_{j}^{\prime}$.

For the case when $j=n$ we can easily show that there is an element of $F$ separable from a set with at least $\lceil n / 2\rceil$ elements of $F$; in the remainder of this section we assume that $j<n$.
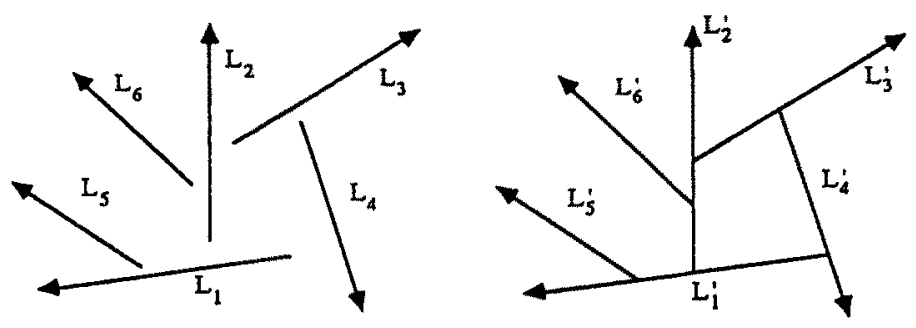

Fig. 5 
For every $i=2, \ldots, j$ let $S_{i}$ be the subset of $F^{\prime}$ consisting of $L_{i}^{\prime}$ together with all the elements of $F^{\prime}$ contained in the open region bounded by $L_{i}^{\prime}$ and $L_{i-1}^{\prime}$ and let $S_{1}$ be the subset of $L^{\prime}$ consisting of $L_{1}^{\prime}$ and all elements of $F^{\prime}$ contained in the open region bounded by $L_{1}^{\prime}$ and $L_{j}^{\prime}$.

Let $i$ be the smallest index such that the line $L$ supporting $L_{1}^{\prime}$ intersects $L_{i}^{\prime}$. Then it is easy to see that the set $A=S_{2} \cup \cdots \cup S_{i-1}$ is separable from $L_{1}$. It is also easy to see that $B=S_{i}$ is separable from $L_{i-1}^{\prime}$ and that

$$
C=S_{i+1} \cup \cdots \cup S_{j} \cup S_{1}
$$

may be separated from $L_{i}^{\prime}$ (see Fig. 6).

However, since $A \cup B \cup C=F^{\prime}$, at least one of them has $\lfloor n / 3\rfloor$ elements; moreover, if not all their cardinalities are the same, then at least one of them has $\lfloor n / 3\rfloor+1$ elements and the result is proved. Assume then that $A, B$, and $C$ have the same cardinality. Since $j<n$, then at least one of the sets $S_{i}$, without loss of generality say $S_{1}$, contains more than one element $L_{a}^{\prime} \in S_{1}, L_{a}^{\prime} \neq L_{1}^{\prime}$. It is now easy to see that $L_{a}$ is separable from $A \cup\left\{L_{1}^{\prime}\right\}$.

\section{Conclusions}

The segments in the example $J_{m}$ may be extended to semilines in such a way that they remain pairwise disjoint. This shows that the bound in Theorem 3 is tight. We think that the $\lceil(n-7) / 4\rceil$ lower bound given in Theorem 1 should be improved to something close to $n / 2$. Like Alon et al. some of us believe that in any family $F$ of $n$ disjoint line segments there is one that can be separated from considerably more than $\lceil(n-1) / 4\rceil$; perhaps from close to $n / 3$ segments. Unlike them, some of us think that the $\lceil(n-1) / 4\rceil$ bound cannot be substantially improved.

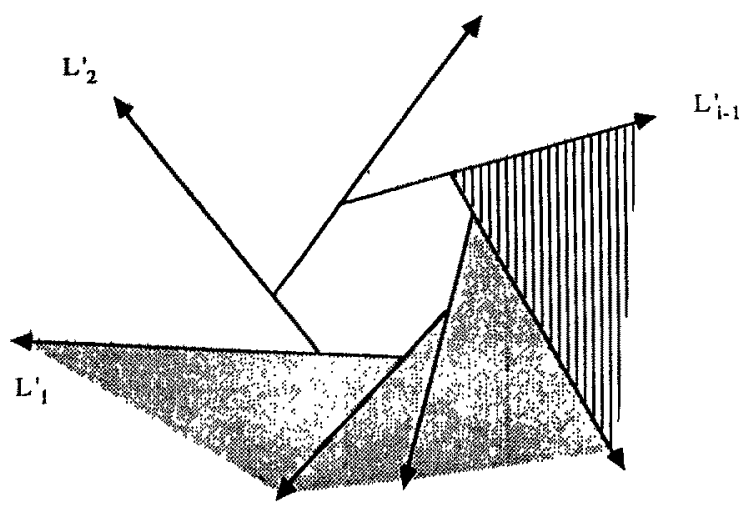

Region separable from $L_{1}$.

[IIIIIIII Region separable from $L_{\mathrm{i}-1}$.

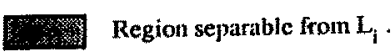

Fig. 6 


\section{References}

1. N. Alon, M. Katchalski, and W. R. Pulleyblank. Cutting disjoint disks by straight lines, Discrete Comput. Geom. 4 (1989), 239-243.

2. K. Hope and M. Katchalski, Separating plane convex sets, Math. Scand., 66 (1990), 44-46.

3. H. Tverberg, A separation property of plane convex sets, Math. Scand. 45 (1979), 255-260.

Received November 27, 1989, and in revised form June 22, 1990. 